\title{
Reduced Regional Gray Matter Volume in Patients with Chronic Obstructive Pulmonary Disease: A Voxel-Based Morphometry Study
}

\author{
H. Zhang, X. Wang, J. Lin, Y. Sun, Y. Huang, T. Yang, S. Zheng, M. Fan, and J. Zhang
}

\section{ABSTRACT}

BACKGROUND AND PURPOSE: Decreased oxygen supply may cause neuronal damage in the brains of patients with COPD, which is manifested by clinical symptoms such as neuropsychological deficits and mood disorders. The aim of the present study was to investigate brain gray matter change in COPD.

MATERIALS AND METHODS: Using voxel-based morphometry based on the high-resolution 3D T1-weighted MR images of GM volume, we investigated 25 stable patients with COPD and 25 matching healthy volunteers. A battery of neuropsychological tests was also performed.

RESULTS: Patients with COPD (versus controls) showed reduced GM volume in the frontal cortex (bilateral gyrus rectus, bilateral orbital and inferior triangular gyri, and left medial superior gyrus), right anterior insula, cingulate cortex (left anterior and middle gyri, right middle gyrus), right thalamus/pulvinar, right caudate, right putamen, right parahippocampus, and left amygdala. In COPD, in some of these regions, regional $\mathrm{GM}$ volume had positive correlations with arterial blood $\mathrm{po}_{2}$, while in some regions, regional $\mathrm{GM}$ volume had negative correlations with disease duration. Patients with COPD (versus controls) had poorer performance in the Mini-Mental State Examination, Visual Reproduction, and Figure Memory tests. Moreover, the GM volume in the inferior triangular frontal cortex in patients with COPD was significantly correlated with the Picture Memory score.

CONCLUSIONS: Our findings suggest GM reductions in a number of brain regions in COPD, which were associated with disease severity and may underlie the pathophysiologic and psychological changes in patients with COPD.

ABBREVIATIONS: COPD = chronic obstructive pulmonary disease; DARTEL = Diffeomorphic Anatomical Registration Through Exponentiated Lie algebra; FEV = forced expiratory volume; $F E V 1=$ forced expiratory volume in 1 second; $F V C=$ forced vital capacity; $G M=$ gray matter; $\mathrm{SaO}_{2}=$ arterial oxyhemoglobin saturation

OPD continues to be a major cause of death. ${ }^{1}$ The irreversible airflow limitation characteristic of COPD is usually progressive, which could subsequently cause decreased oxygen supply to

Received March 29, 2012; accepted after revision May 23.

From the Department of Physiology and Neurobiology (H.Z., Y.S., Y.H., J.Z.), Medical College of Xiamen University, Xiamen, China; Department of Neurology (X.W.), Affiliated Hospital of Hangzhou Normal University, Hangzhou, China; Magnetic Resonance Center (J.L., T.Y.) and Department of Respiratory Medicine (S.Z.), Zhongshan Hospital, Medical College of Xiamen University, Xiamen, China; and Department of Brain Protection and Plasticity (M.F.), Institute of Basic Medical Sciences, Beijing, China.

H.Z. and X.W. contributed equally to this work.

Authors' contributions: Haiyan Zhang contributed to the study design and took responsibility for the integrity of the data and the accuracy of the data analysis. Xiaochuan Wang contributed to the study design, collection, analysis, interpretation of data, and critical review. Jianzhong Lin contributed to the collection, analysis, and interpretation of data. Yingchun Sun contributed to the collection, analysis, and interpretation of data. Yongxia Huang contributed to the collection, analysis, and interpretation of data. Tianhe Yang contributed to the collection, analysis, and interpretation of data. Shili Zheng contributed to the collection, analysis, and interpretation of data. Ming Fan contributed to the study design, drafting, critical review, and final approval of the manuscript. Jiaxing Zhang contributed to conception and design, interpretation of data, drafting the article, and final approval of the manuscript. the brain. Because the brain is extremely sensitive to changes in arterial oxygen concentration, ${ }^{2}$ it inevitably has hypoxic stress. In addition, patients with COPD often have systemic inflammation, ${ }^{1}$ which may exacerbate neuronal injury. In fact, neuronal damage in the brains of patients has been manifested in clinical symptoms such as neuropsychological deficits, ${ }^{2-6}$ depression and anxiety, ${ }^{1,7}$ and physical disabilities. ${ }^{1}$ Deterioration in cerebral perfusion $^{8}$ and a decrease in cerebral metabolites ${ }^{9-12}$ have also been reported. All the above characteristics of COPD suggest the presence of brain structure alteration. However, until now, it remains largely uninvestigated, to our knowledge.

This work was supported by the National Science Foundation of China (81181324) National Science Foundation of China (31071041), Bureau of Science and Technology of Hangzhou (20090833B10), and Health Bureau of Zhejiang Province (2009A168). We certify that there is no conflict of interest with any financial organization regarding the material discussed in the article.

Please address correspondence to Jiaxing Zhang, MD, Department of Physiology and Neurobiology, Medical College of Xiamen University, Xiamen, 361005, China; e-mail: zhangjiaxing@xmu.edu.cn

- Indicates open access to non-subscribers at www.ajnr.org

http://dx.doi.org/10.3174/ajnr.A3235 
In this study, voxel-based morphometry ${ }^{13}$ based on 3D T1weighted MR imaging, was used to measure GM volume in patients with COPD. Recently, the preprocessing steps of voxelbased morphometry have been improved with the DARTEL registration method. ${ }^{14}$ The DARTEL algorithm uses a more sophisticated mathematic framework and achieves sharper nonlinear registration than parametric approaches, which exponentiates a velocity field to produce deformations. A "diffeomorphism" is a globally one-to-one (objective) smooth and continuous mapping with derivatives that are invertible. This is an important advance for transformation to best represent the normalization of a brain image to an appropriate template. Therefore, instead of as previously simply matching a pair of images, DARTEL can achieve accurate realignment of small inner structures. Voxel-based morphometry DARTEL has been widely applied in clinical studies. ${ }^{15}$

Functional MR imaging studies on breathlessness, air hunger, and inspiratory-loaded breathing have revealed that a large number of brain regions, including the frontal cortices, temporal cortices, limbic/paralimbic cortices, cerebellum, and midbrain, are activated by dyspnea. ${ }^{16}$ Moreover, these dyspnea-activated brain regions have also been shown to be impaired in patients with long-term sustained hypoxia exposure, such as patients with congenital central hypoventilation syndrome ${ }^{17}$; those with long-term intermittent hypoxia exposure, such as obstructive sleep apnea ${ }^{18,19}$; and high-altitude residents. ${ }^{20}$ Dyspnea is the most common and disabling symptom in patients with COPD. We therefore hypothesized that patients with COPD would have similar cerebral impairment.

\section{MATERIALS AND METHODS \\ Subjects}

Twenty-five patients were enrolled from December 2009 to May 2011. All patients had undergone a period of 30-45 days of inhospital rehabilitation following an acute exacerbation of COPD. At the time of data collection, patients were in a stable condition. Among these patients, 12 discharged patients were recruited during their rest at home and 13 patients were recruited when they were awaiting discharge from the hospital. Patients were diagnosed in Zhongshan Hospital (Xiamen, China) according to the diagnostic criteria of the Global Initiative for Chronic Obstructive Lung Disease. ${ }^{21}$ Twenty-five healthy volunteers, with comparable age, sex, and educational background, composed the control group. All subjects were free from a known history of cerebrovascular accident, heart failure, neurologic disorders, obstructive sleep apnea, or other diseases known to affect cognition. Patients were provided with therapy, including inhaled ipratropium bromide, bricanyl, albuterol (Ventolin), and budesonide. Demographic characteristics of the patients and healthy volunteers are listed in Table 1. All subjects provided written informed consent. The experimental protocol was approved by the Research Ethics Review Board of Xiamen University.

\section{Physiologic and Neuropsychological Tests}

Physiologic and neuropsychological tests and assessment of activities of daily living (score range, 14-56) ${ }^{22}$ were conducted 1 day
Table 1: Demographic characteristics of the patients with COPD and healthy volunteers

\begin{tabular}{lccr}
\hline & $\begin{array}{c}\text { Patients } \\
\text { with COPD }\end{array}$ & Controls & $\begin{array}{c}\boldsymbol{P} \\
\text { Value }\end{array}$ \\
\hline No. of subjects & 25 & 25 & \\
Sex (female) (\%) & 16 & 16 & \\
Age (yr) (mean) & $69.2 \pm 8.1(58-84)$ & $68.0 \pm 8.0(57-86)$ & .59 \\
Education (yr) (mean) & $6.7 \pm 3.9$ & $7.5 \pm 5.0$ & .53 \\
Family history of & 4 & - & \\
$\quad$ COPD (\%) & & & \\
Disease duration (yr) & $7.0 \pm 5.7$ & - & \\
Actual smokers (\%) & 44 & 40 & .86 \\
\hline
\end{tabular}

before the MR imaging. Physiologic tests included pulse rate and arterial blood pressure measures, arterial blood gas analysis, and pulmonary function measures. Blood samples were taken in the morning between 7:00 and 7:30. The neuropsychological tests included the following: 1) the Chinese version of the Mini-Mental State Examination, which measures general cognitive function; and 2) the Visual Reproduction Test, Figure Memory Test, and Digit Span Forward and Backward Tasks, which, taken from the Chinese revised version of the Wechsler Memory Scale, were used to measure visuoconstructive ability, visuospatial memory, shortterm verbal memory, and working memory, respectively. All data were analyzed by using the Statistical Package for the Social Sciences, Version 19.0 (SPSS, Chicago, Illinois). An independent $t$ test measured between-group differences. Statistical significance was set at $P<.05$.

\section{MR Imaging Data Acquisition}

Images were acquired on a Tim Trio 3T scanner (Siemens, Erlangen, Germany) at the MR Imaging Center (Zhongshan Hospital, Xiamen, China). 3D structural MR imaging was performed in each subject by using a T1-weighted magnetization-prepared rapid acquisition of gradient echo sequence $(\mathrm{TR} / \mathrm{TE}=1900 / 2.48$ $\mathrm{ms}, \mathrm{FOV}=25 \times 25 \mathrm{~cm}^{2}, \mathrm{NEX}=1$, matrix $=512 \times 256$, section thickness $=1.0 \mathrm{~mm}$ ). Conventional 2D T1 and T2 images were also acquired and examined for any incidental findings. The data analysis was conducted by 2 researchers who were blinded to the status of subjects.

\section{Voxel-Based Morphometry Analysis}

Data were analyzed by using the VBM8 toolbox implemented in SPM8 (Wellcome Department of Imaging Neuroscience, University College London, London, UK) ${ }^{13}$ Calculations and image matrix manipulations were performed by using Matlab (MathWorks, Natick, Massachusetts). The processes included the following steps: 1) the images were inspected for artifacts and set at the anterior commissure. Using the unified segmentation procedure, we segmented the reorientated image into GM, white matter, and CSF in the same space as the original T1-weighted image. Procrustes-aligned GM images were generated by a rigidbody transformation, which used isomorphic scaling, translation, and rotation to find the "best" fit between $\geq 2$ landmarked shapes. ${ }^{13}$ 2) The DARTEL registration method, which is implemented in SPM8, was used to create a study-specific template by using the aligned images from all patients and controls to improve intersubject registration of structural images. ${ }^{14}$ The procedure involved 6 iterations, which began with creating an initial tem- 
Table 2: Physiologic and psychological characteristics ${ }^{\text {a }}$

\begin{tabular}{|c|c|c|c|}
\hline & $\begin{array}{c}\text { Patients } \\
\text { with COPD }\end{array}$ & Controls & $\begin{array}{c}P \\
\text { Value }\end{array}$ \\
\hline BMI $\left(\mathrm{kg} / \mathrm{m}^{2}\right)$ & $20.8 \pm 3.9$ & $22.6 \pm 2.6$ & .081 \\
\hline ADL & $20.1 \pm 6.5$ & $14.6 \pm 1.5$ & $<.001$ \\
\hline Heart rate & $92.4 \pm 16.2$ & $70.9 \pm 8.9$ & $<.001$ \\
\hline \multicolumn{4}{|l|}{ Blood pressure $(\mathrm{mm} \mathrm{Hg})$} \\
\hline Systolic pressure & $136.1 \pm 19.1$ & $136.5 \pm 18.0$ & .940 \\
\hline Diastolic pressure & $81.8 \pm 10.7$ & $77.5 \pm 14.5$ & .307 \\
\hline \multicolumn{4}{|l|}{ Hematologic measurements } \\
\hline $\mathrm{SaO}_{2}(\%)$ & $94.0 \pm 4.2$ & $97.0 \pm 1.3$ & .003 \\
\hline $\mathrm{po}_{2}(\mathrm{~mm} \mathrm{Hg})$ & $79.9 \pm 23.3$ & $98.5 \pm 11.3$ & .006 \\
\hline $\mathrm{pCO}_{2}(\mathrm{MM} \mathrm{HG})$ & $48.1 \pm 6.0$ & $39.8 \pm 3.0$ & $<.001$ \\
\hline $\mathrm{pH}$ & $7.4 \pm 0.06$ & $7.4 \pm 0.01$ & .731 \\
\hline \multicolumn{4}{|l|}{ Pulmonary function testing } \\
\hline Respiratory rate (breaths/min) & $23.5 \pm 6.0$ & $16.9 \pm 5.5$ & .003 \\
\hline FVC (\% predicted) & $66.6 \pm 17.2$ & $96.1 \pm 14.7$ & $<.001$ \\
\hline FEV1 (\% predicted) & $43.4 \pm 16.4$ & $97.5 \pm 16.9$ & $<.001$ \\
\hline FEV1/FVC (\%) & $50.3 \pm 10.7$ & $80.0 \pm 8.3$ & $<.001$ \\
\hline \multicolumn{4}{|l|}{ Cognitive tests } \\
\hline MMSE & $23.3 \pm 3.4$ & $25.8 \pm 2.1$ & .021 \\
\hline \multicolumn{4}{|l|}{ Digit Span } \\
\hline Forward Task & $7.0 \pm 1.6$ & $7.7 \pm 1.4$ & 0.125 \\
\hline Backward Task & $4.1 \pm 1.9$ & $4.3 \pm 1.5$ & 0.612 \\
\hline Visual Reproduction & $8.2 \pm 3.4$ & $10.3 \pm 3.0$ & 0.031 \\
\hline Figure Memory & $10.5 \pm 3.0$ & $12.4 \pm 1.9$ & 0.010 \\
\hline
\end{tabular}

Note:-ADL indicates activities of daily living; BMI, body mass index; MMSE, MiniMental State Examination; $\mathrm{PCO}_{2}=$ partial pressure of carbon dioxide.

${ }^{\text {a }}$ Data are mean $\pm S D$.

plate from all the aligned images, and was followed by the registration on each of the subjects in turn. Then, the first iteration of the registration was completed in each subject, and a new template was created. After this, the second iteration began. When 6 iterations of averaging and registration were finished, the final template was generated, which was the average of the DARTEL registered data. During iterations, all images were warped to the template, yielding a series of flow fields that parameterized deformations to use in modulations to preserve actual GM volume. The GM images were modulated to account for the local compression and stretching that occurs as a consequence of the warping and affine transformation, which is based on the change of variables theorem. 3) The normalized images were transformed into Montreal Neurological Institute space. These GM images were then smoothed by using a Gaussian kernel of $8-\mathrm{mm}$ full width at half maximum. Independent $t$ tests were performed to examine between-group differences. The statistical parametric map was generated with the voxel level threshold at $t>3.7473, P<.05$ (false discovery rate correction with sex, age, and education as covariates). False discovery rate correction was used because it focuses on the proportion of rejections that are false. It is less conservative and easy to interpret, and it offers an objective and data-driven perspective for threshold statistical maps. ${ }^{23}$

To analyze the correlation of GM image values with cognitive or physiologic measurement, we took the following steps: 1) Regions of interest were created for clusters showing differences between groups, and 2) by using these region-of-interest masks, the GM values were extracted from each individual's normalized and smoothed GM maps. Then the partial correlations were analyzed by using SPSS. Statistical significance was set at $P<.05$, with sex, age, and education as covariates.

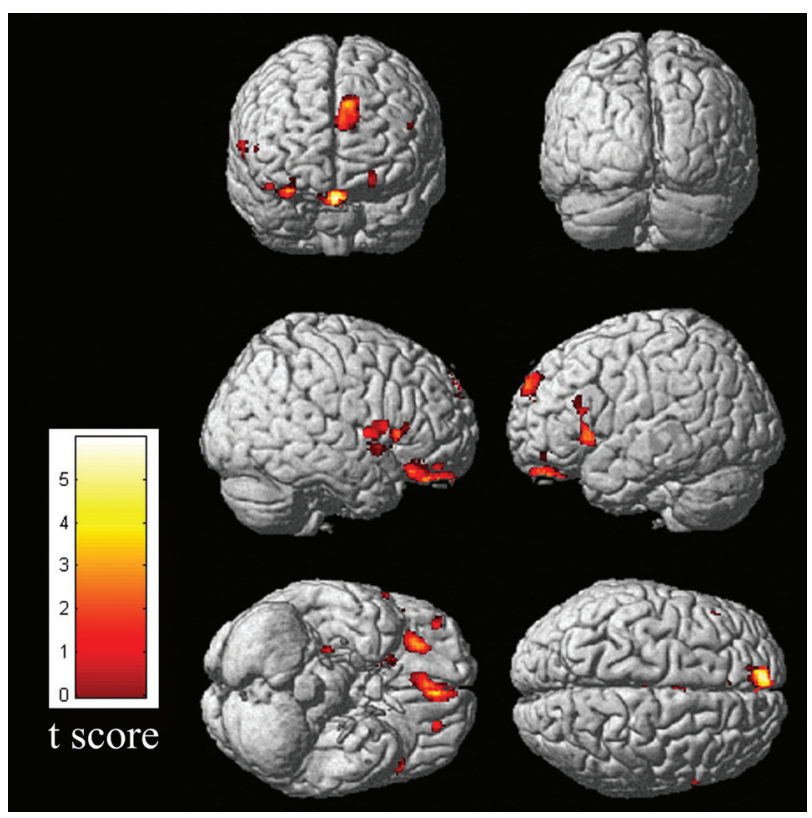

FIG 1. Results of voxel-based morphometry-DARTEL analysis of the entire group of patients with COPD versus the entire group of controls. Note all regions in which patients with COPD had a significantly decreased gray matter volume compared with the controls at $P<.05$, corrected for multiple comparisons. Maximum intensity projections of all areas with significantly reduced gray matter volumes in the statistical parametric mapping (SPM) group analysis as an overlay on the SPM standard brain template.

\section{RESULTS}

\section{Physiologic and Behavioral Findings}

Compared with the controls, patients with COPD had markedly lower arterial blood $\mathrm{SaO}_{2}$ and $\mathrm{pO}_{2}$ values and higher arterial blood $\mathrm{pCO}_{2}$ values and heart rates (Table 2). Patients with COPD had markedly lower 1-second FVC, FEV, and FEV1/FVC values and higher respiratory rates. Patients with COPD had markedly lower scores in the activities of daily life, the Mini-Mental State Examination, and Visual Reproduction, and Figure Memory tests.

\section{Regional GM Volume}

No subject from either group showed visible abnormalities on T1-weighted structural images. Voxel-based morphometry analysis showed that patients with COPD had reduced regional GM volumes compared with healthy controls in the frontal cortex (bilateral gyrus rectus, bilateral orbital gyrus, bilateral inferior triangular gyrus, and left medial superior gyrus), right anterior insula, cingulate cortex (left anterior and middle gyri, right middle gyrus), right thalamus/pulvinar, right caudate, right putamen, right parahippocampus, and left amygdala (cluster size, $>70$ voxels) (Figs 1-4).

\section{Correlations}

In patients with COPD, partial correlation (controlling for disease duration, FEV1/FVC, and age) revealed that GM volume in the gyrus rectus $(r=0.531, P=.017)$, right frontal inferior orbital gyrus ( $r=0.496, P=.025)$, left frontal superior orbital gyrus ( $r=$ $0.459, P=.037)$, right insula $(r=0.564, P=.012)$, right middle cingulate gyrus $(r=0.490, P=.027)$, right thalamus/pulvinar $(r=0.746, P<.001)$, and left amygdala $(r=0.471, P=.033)$ had positive correlations with arterial blood $\mathrm{pO}_{2}$ values. Partial corre- 


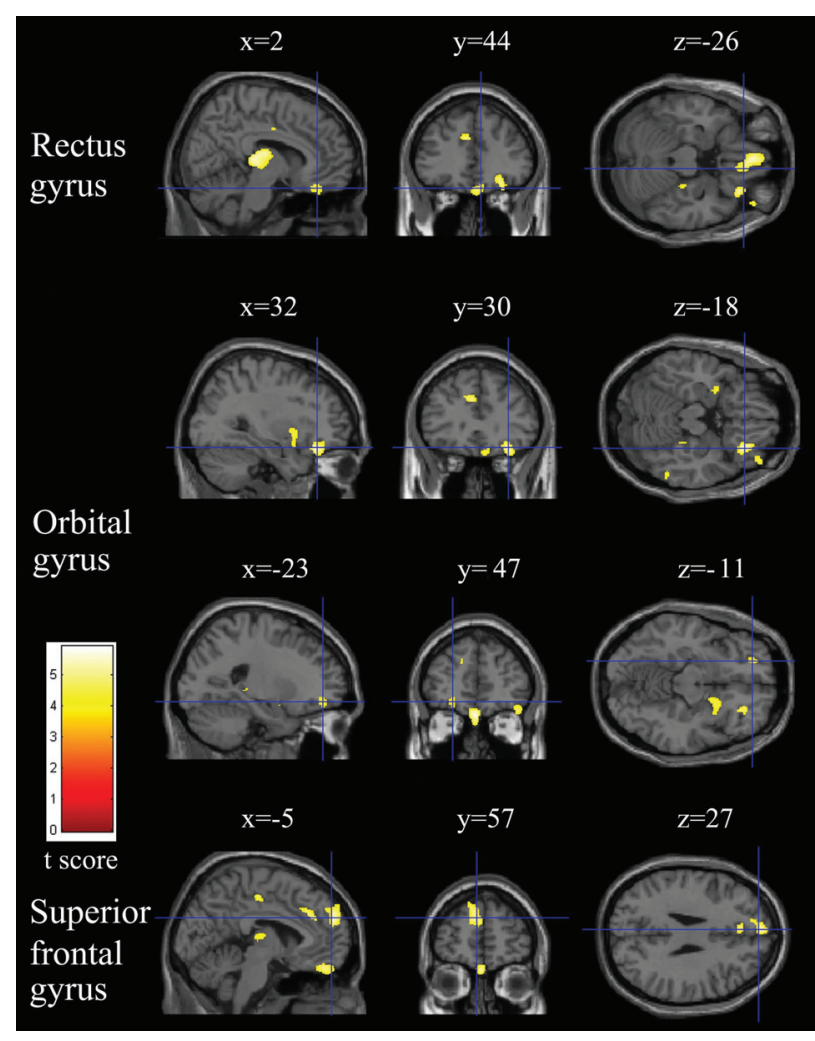

FIG 2. Relatively reduced clusters (yellow) in the gray matter in patients with COPD compared with healthy controls $(P<.05$, false discovery rate-corrected). Sections (sagittal, coronal, and axial view) show reduced gray matter volume in the right gyrus rectus, bilateral orbital gyrus, and left superior frontal gyrus overlaid on a T7-weighted anatomic MR image in the Montreal Neurological Institute template.

lation (controlling for $\mathrm{pO}_{2}, \mathrm{FEV} 1 / \mathrm{FVC}$, and age) revealed that GM volume in the right frontal inferior orbital gyrus $(r=-0.463, P=$ $.015)$, right inferior triangular gyrus $(r=-0.436, P=.021)$, left medial superior frontal gyrus $(r=-0.417, P=.027)$, right insula $(r=-0.623, P=.001)$, and right parahippocampal gyrus $(r=$ $-0.564, P=.003$ ) had negative correlations with disease duration. GM volume in the right inferior triangular frontal gyrus in patients with COPD correlated significantly with the Picture Memory score $(r=0.475, P=.019)$.

\section{DISCUSSION}

Decreased oxygen supply, resulting from the irreversible airflow limitation, may cause neuronal damage in the brains of patients with COPD. Our present study revealed that patients with COPD had reduced GM volume in a number of brain regions, which were mainly confined to the limbic/paralimbic structures and frontal cortices, compared with healthy controls. These findings are consistent with those in patients with obstructive sleep apnea, ${ }^{18,19,24}$ congenital central hypoventilation syndrome, and pediatric heart failure, ${ }^{25}$ and in high-altitude residents. ${ }^{20}$ Moreover, patients with COPD exhibited poorer performance on visuospatial and visual reproduction tasks.

GM volume in the reduced regions in patients with COPD had a strongly positive correlation with arterial blood $\mathrm{pO}_{2}$ values, which suggested that the reduced GM may result from sustained low blood oxygen. Hypoxia-induced metabolic decreases ${ }^{9,10,12}$

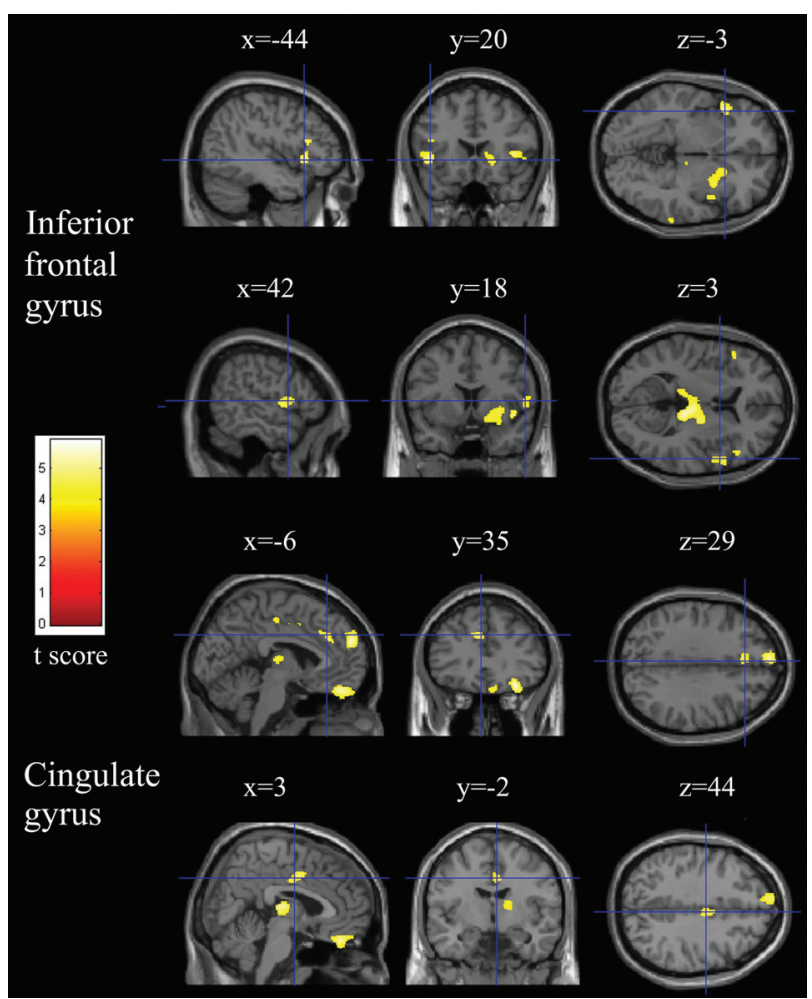

FIG 3. Relatively reduced clusters (yellow) in gray matter in patients with COPD compared with healthy controls $(P<.05$, false discovery rate-corrected). Sections (sagittal, coronal, and axial view) show reduced gray matter volume in the bilateral inferior frontal and bilateral cingulate gyri overlaid on a T1-weighted anatomic MR image in the Montreal Neurological Institute template.

and cerebral perfusion decline $e^{8,26}$ in COPD may be the cause of morphologic reductions. In addition, systemic inflammation in COPD can exacerbate neuronal injury. ${ }^{1}$ A greater proportion of regions showing GM loss located in the limbic/paralimbic cortices in patients with COPD found in the present study may be due to the fact that those phylogenetically older regions of the brain showed sharper vascular responses to hypoxia than evolutionarily younger regions. ${ }^{27}$ Although there is evidence that cigarette smoking may cause neuronal damage in the brains of patients with COPD, nicotine has no selective effect on some brain regions. In our study, we did not detect a correlation of the duration or the amount of smoking with the regional GM volume among all smokers. Moreover, further analysis revealed that healthy control smokers had normal regional GM volume compared with the patients with COPD who smoked and compared with the healthy control non-smokers. Regardless, a further study should be conducted, increasing the number of subjects, to investigate more clearly the effect of smoking on brain structures.

Various studies have suggested that the unpleasantness of subjectively perceived dyspnea was processed in distinct brain areas. However, neuroimaging studies have suggested that the right anterior insula seemed to be the most consistent structure across studies. ${ }^{16}$ Therefore, we speculated that the reduction in the right anterior insular GM would have reduced the perception of dyspnea in patients with COPD. The finding that patients with insula lesions exhibit a decrease in unpleasant pain, sharpness, and thermal sensation may support our assumption. ${ }^{28}$ In addition, mor- 


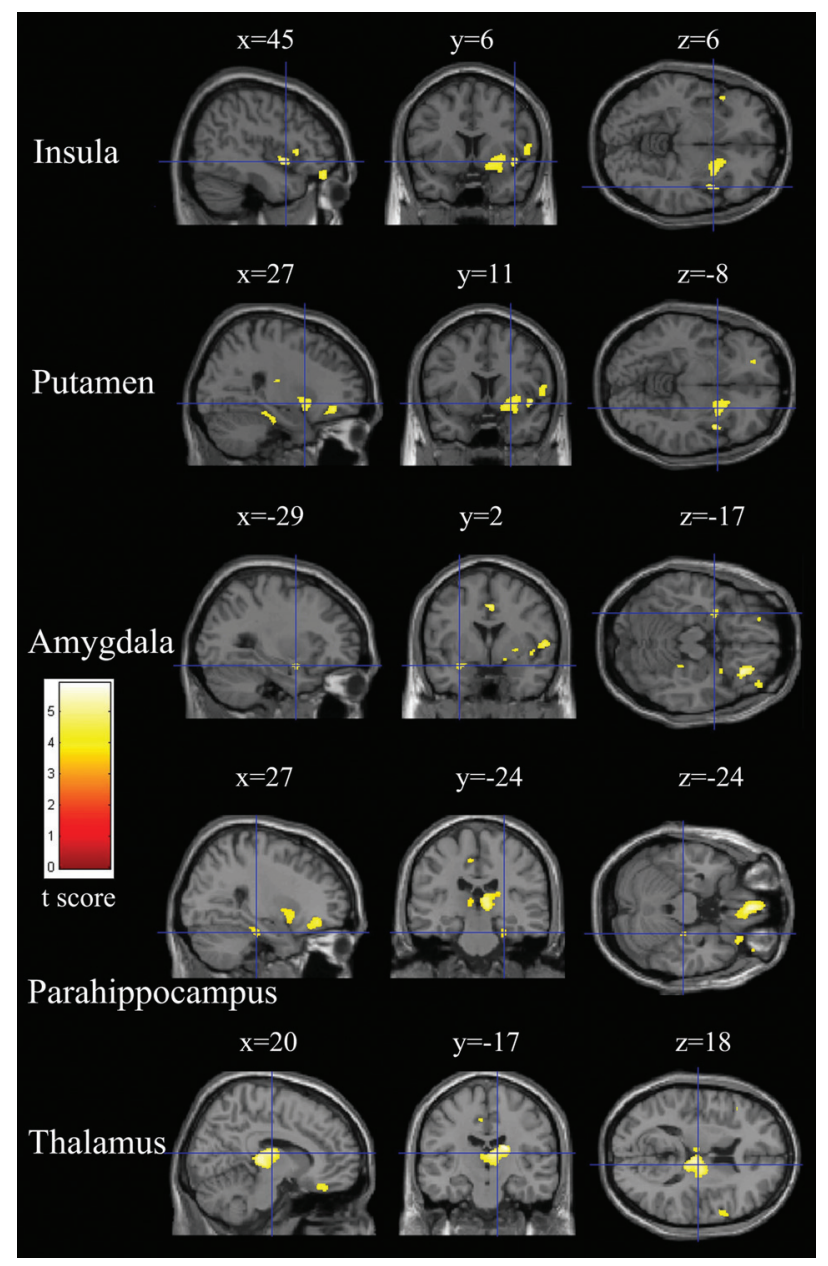

FIG 4. Relatively reduced clusters (yellow) in gray matter in patients with COPD compared with healthy controls $(P<.05$, false discovery rate-corrected). Sections (sagittal, coronal, and axial view) show reduced gray matter volume in the right anterior insula, right putamen, left amygdala, right parahippocampus, and right thalamus overlaid on a T1-weighted anatomic MR image in the Montreal Neurological Institute template.

phologic reductions in the anterior cingulate cortex could also play a role in the perception of air hunger because it, together with the anterior insula, constitutes a neural substrate for the control and suppression of physiologic urges. ${ }^{29}$ The reduced GM in the posterior thalamus may clarify the enhanced breathing movements in patients with COPD. Lesions in the posterior thalamus abolished hypoxia-induced inhibition of fetal breathing movements, while local electrical stimulation effectively decreased respiratory frequency. ${ }^{30}$ Reduced GM in the right insula may explain cardiovascular disturbances in patients with COPD. ${ }^{1}$ Considerable evidence suggests that the insula, especially the right insula, is implicated in cardiac rate and rhythm control. ${ }^{31}$ The insula regulates cardiovascular activity through connecting with the components of limbic systems, including the anterior cingulate gyrus. ${ }^{32}$ A previous study has shown that the insula contributes to the long-lasting changes in cardiac rate in response to hypoxia. ${ }^{33}$

In the present study, patients with COPD had poorer performance in general cognitive function, visuospatial memory, and visuoconstructive tasks; these findings were consistent with pre- vious ones. ${ }^{3-5}$ The GM volume in the right inferior triangular frontal gyrus has a positive correlation with the Picture Memory score, suggesting that it may be responsible for the deficits in visuospatial tasks. Previous studies have suggested that the right inferior frontal cortex processes emotional communicative information with visual input during the observation and execution of actions. $^{34,35}$ The reduced GM volume in the anterior insular cortex, anterior cingulate cortex, and pulvinar may contribute to the deficits in visuoconstructive tasks. Our assumption was based on the following previous data: 1) Visual stimuli activated the network comprising the anterior insular cortex, anterior cingulate cortex, and prefrontal cortex, ${ }^{36}$ and 2) the pulvinar has connections to the visual areas and to the frontal and parietal oculomotor cortices and has been implicated in various visual functions in lesion studies. ${ }^{37}$

\section{CONCLUSIONS}

We first demonstrated that COPD extended to the brain, with the reduction of regional GM volume in a number of brain regions. Our findings suggest significant participation of these structures in responding to hypoxic challenges, which include cardiovascular and air-hunger components. The brain structural changes may also underlie the psychological and mood changes in patients with COPD.

\section{REFERENCES}

1. Barnes PJ. Chronic obstructive pulmonary disease: effects beyond the lungs. PLoS Med 2010; 7:e1000220

2. Erecińska M, Silver IA. Tissue oxygen tension and brain sensitivity to hypoxia. Respir Physiol 2001;128:263-76

3. Incalzi RA, Gemma A, Marra C, et al. Chronic obstructive pulmonary disease: an original model of cognitive decline. Am Rev Respir Dis 1993; 148:418-24

4. Antonelli-Incalzi R, Corsonello A, Pedone C, et al. Drawing impairment predicts mortality in severe COPD. Chest 2006;130:1687-94

5. Ozge $\mathrm{C}$, Ozge A, Unal O. Cognitive and functional deterioration in patients with severe COPD. Behav Neurol 2006;17:121-30

6. Hung WW, Wisnivesky JP, Siu AL, et al. Cognitive decline among patients with chronic obstructive pulmonary disease. Am J Respir Crit Care Med 2009;180:134-37

7. Schneider C, Jick SS, Bothner U, et al. COPD and the risk of depression. Chest 2010;137:341-47

8. Ortapamuk H, Naldoken S. Brain perfusion abnormalities in chronic obstructive pulmonary disease: comparison with cognitive impairment. Ann Nucl Med 2006;20:99-106

9. Shim TS, Lee JH, Kim SY, et al. Cerebral metabolic abnormalities in COPD patients detected by localized proton magnetic resonance spectroscopy. Chest 2001;120:1506-13

10. Sicard KM, Duong TQ. Effects of hypoxia, hyperoxia, and hypercapnia on baseline and stimulus-evoked BOLD, CBF, and $\mathrm{CMRO} 2$ in spontaneously breathing animals. Neuroimage 2005;25:850-58

11. Borson S, Scanlan J, Friedman S, et al. Modeling the impact of COPD on the brain. Int J Chron Obstruct Pulmon Dis 2008;3:429-34

12. Sinha S, Kumar V, Jagannathan NR, et al. Proton magnetic resonance spectroscopy of brain to study the cerebral metabolic abnormalities in COPD patients: a case control study in north India. Indian J Chest Dis Allied Sci 2009;51:15-19

13. Ashburner J, Friston KJ. Voxel-based morphometry-the methods Neuroimage 2000;11:805-21

14. Ashburner J. A fast diffeomorphic image registration algorithm. Neuroimage 2007;38:95-113

15. Chen Z, Li L, Sun J, et al. Mapping the brain in type II diabetes: 
voxel-based morphometry using DARTEL. Eur J Radiol 2012;81: $1870-76$

16. von Leupoldt A, Sommer T, Kegat S, et al. The unpleasantness of perceived dyspnea is processed in the anterior insula and amygdala. Am J Respir Crit Care Med 2008;177:1026-32

17. Macey PM, Richard CA, Kumar R, et al. Hippocampal volume reduction in congenital central hypoventilation syndrome. PLoS One 2009;4:e6436

18. Macey PM, Henderson LA, Macey KE, et al. Brain morphology associated with obstructive sleep apnea. Am J Respir Crit Care Med 2002;166:1382-87

19. Joo EY, Tae WS, Lee MJ, et al. Reduced brain gray matter concentration in patients with obstructive sleep apnea syndrome. Sleep 2010;33:235-41

20. Zhang J, Yan X, Shi J, et al. Structural modifications of the brain in acclimatization to high-altitude. PLoS One 2010;5:e11449

21. Pauwels RA, Buist AS, Calverley PM, et al. Global strategy for the diagnosis, management, and prevention of chronic obstructive pulmonary disease: NHLBI/WHO Global Initiative for Chronic Obstructive Lung Disease (GOLD) Workshop summary. Am J Respir Crit Care Med 2001;163:1256-76

22. Lawton MP, Brody EM. Assessment of older people: self-maintaining and instrumental activities of daily living. Gerontologist 1969;9:179-86

23. Chen S, Wang C, Eberly LE, et al. Adaptive control of the false discovery rate in voxel-based morphometry. Hum Brain Mapp 2009;30:2304-11

24. Torelli F, Moscufo N, Garreffa G, et al. Cognitive profile and brain morphological changes in obstructive sleep apnea. Neuroimage 2011;54:787-93

25. Menteer J, Macey PM, Woo MA, et al. Central nervous system changes in pediatric heart failure: a volumetric study. Pediatr Cardiol 2010;31:969-76
26. Nöth U, Kotajima F, Deichmann R, et al. Mapping of the cerebral vascular response to hypoxia and hypercapnia using quantitative perfusion MRI at 3 T. NMR Biomed 2008;21:464-72

27. Binks AP, Cunningham VJ, Adams L, et al. Gray matter blood flow change is unevenly distributed during moderate isocapnic hypoxia in humans. J Appl Physiol 2008;104:212-17

28. Bowsher D. Somatic sensation and the insular-opercular cortex: relationship to central pain. Eur Neurol 2006;55:160-65

29. Lerner A, Bagic A, Hanakawa T, et al. Involvement of insula and cingulate cortices in control and suppression of natural urges. Cereb Cortex 2009; 19:218-23

30. Teppema LJ, Dahan A. The ventilatory response to hypoxia in mammals: mechanisms, measurement, and analysis. Physiol Rev 2010;90:675-754

31. Oppenheimer S. Cerebrogenic cardiac arrhythmias: cortical lateralization and clinical significance. Clin Auton Res 2006;16:6-11

32. Nagai M, Hoshide S, Kario K. The insular cortex and cardiovascular system: a new insight into the brain-heart axis. J Am Soc Hypertens 2010;4:174-82

33. Macey PM, Valderama C, Kim AH, et al. Temporal trends of cardiac and respiratory responses to ventilatory challenges in congenital central hypoventilation syndrome. Pediatr Res 2004;55:953-59

34. Gallese V, Fadiga L, Fogassi L, et al. Action recognition in the premotor cortex. Brain 1996;119(pt 2):593-609

35. Nakamura K, Kawashima R, Ito K, et al. Activation of the right inferior frontal cortex during assessment of facial emotion. J Neurophysiol 1999;82:1610-14

36. Sterzer $\mathrm{P}$, Kleinschmidt A. Anterior insula activations in perceptual paradigms: often observed but barely understood. Brain Struct Funct 2010;214:611-22

37. Van der Stigchel S, Arend I, van Koningsbruggen MG, et al. Oculomotor integration in patients with a pulvinar lesion. Neuropsychologia 2010;48:3497-504 